\title{
EXISTENCE OF POSITIVE HARMONIC FUNCTIONS ${ }^{1}$
}

\section{MITSURU NAKAI}

1. Consider an open Riemann surface $R$. In this note by a distinguished subregion $G$ of $R$ we understand that $G$ is a subregion of $R$ with nonempty analytic relative boundary $\partial G$ and with noncompact closure $\bar{G}$. The purpose of this note is to give a simple proof of the following theorem of Parreau [5]: ${ }^{2}$

TheOREM. For any distingished subregion $G$ of an arbitrary open Riemann surface $R$, there exists a nonconstant positive harmonic function $u$ on $G$ with continuous boundary value zero on $\partial G$.

It is interesting to compare the theorem ${ }^{3}$ with the so-called "two domains criterion" due to Bader-Parreau [1] and Mori [4]: An open Riemann surface $R$ does not belong to the class $O_{H B}$ (resp. $O_{H D}$ ) if and only if there exist two disjoint distinguished subregions of $R$ carrying nonconstant $H B$ (resp. $H D$ ) functions with continuous boundary values zero on their relative boundaries. The theorem shows that the two domains criterion fails for the class $O_{H P}$.

Another consequence of the theorem is that the Martin compactification $G^{*}$ of any distinguished subregion $G$, considered as a Riemann surface, always contains an $H P$-minimal point other than those $H P$-minimal points identified with points in $\partial G$, no matter whether $R \in O_{G}$ or not. For Martin's compactification and $H P$-minimal points see e.g. Constantinescu-Cornea [2].

The proof of the theorem will be given in $\$ \S 2-4$.

2. Let $G$ be a distinguished subregion of an open Riemann surface $R$. Fix a point $p_{0} \in G$. We denote by $g_{G}(p, q)$ the Green's function of $G$. We choose an arbitrary sequence $\left\{q_{n}\right\}$ of points in $G$ converging to the ideal boundary of $G$, i.e. converging to the Alexandroff points of $R$. Following Martin [3] we set

$$
u_{n}(p)=\frac{g_{G}\left(p, q_{n}\right)}{g_{G}\left(p_{0}, q_{n}\right)}
$$

Received by the editors November 10, 1965 and, in revised form, December 13, 1965.

1 This work was sponsored by the U. S. Army Research Office (Durham), Grant DA-ARO(D)-31-124-G 499, University of California, Los Angeles.

2 The author is indebted to Professor K. Oikawa for suggesting this problem to him, and to Professor T. Kuroda for suggesting a reference.

- The thorem can be expressed simply as $G \notin S O_{H P}$. A comparison of this with the following is also interesting: $G \in S O_{H B}$ (resp. $S O_{H D}$ ) if $R \in O_{G}$, and $G \notin S O_{H B}$ (resp. $\left.S O_{H D}\right)$ if $R \notin O_{G}$ and $\partial G$ is compact. 
for $p$ in $R-q_{n}$. Observe that $u_{n}\left(p_{0}\right)=1$. Since $u_{n} \in H P(\Omega)$ for any relatively compact subregion $\Omega$ and for sufficiently large $n,\left\{u_{n}\right\}$ constitutes a normal family. Therefore by chosing a suitable subsequence of $\left\{q_{n}\right\}$, we may assume that

$$
u_{\infty}(p)=\lim _{n \rightarrow \infty} u_{n}(p)
$$

exists in $G$. Obviously $u_{\infty} \in H P(G)$ and $u_{\infty}>0$ in $G$ since $u_{\infty}\left(p_{0}\right)$ $=\lim u_{n}\left(p_{0}\right)=1$.

3. The proof will be complete if we show that $u_{\infty}$ has the continuous boundary value zero on $\partial G$. Take an arbitrary open $\operatorname{arc} \alpha$ in $\partial G$ with compact closure. We only have to show that $u_{\infty}$ has the continuous boundary value zero on $\alpha$.

Join the two endpoints of $\alpha$ by a simple analytic arc $\gamma$ in $G$ so that the subregion $F$ of $G$ bounded by $\alpha \cup_{\gamma}$ is simply connected. By the Riemann mapping theorem we can map $F$ onto the open unit disk $U$ by a conformal mapping $\phi$. By Carathéodory's theorem $\phi$ can be assumed to be a topological mapping of $\bar{F}$ onto $\bar{U}=U \cup C$, where $C$ denotes the unit circle. We set $\beta=\phi(\alpha)$, which is an open subarc of $C$, and

$$
v_{k}(z)=u_{k}\left(\phi^{-1}(z)\right)
$$

on $U$ for $k=1,2, \cdots, \infty$. Clearly $v_{k} \in H P(U)(k=1,2, \cdots, \infty)$ and in view of (2)

$$
v_{\infty}(z)=\lim _{n \rightarrow \infty} v_{n}(z)
$$

on $U$. Moreover $v_{n}$ is continuous on $\bar{U}$ for $n=1,2, \cdots$ and by (1)

$$
v_{n}=0 \quad(n=1,2, \cdots)
$$

on $\beta$. If we show that $v_{\infty}$ has continuous boundary value zero on $\beta$, then the same conclusion follows for $u_{\infty}$ on $\alpha$.

4. Let $\mu_{n}$ be the regular Borel measure on $C$ defined by

$$
d \mu_{n}(\zeta)=\frac{1}{2 \pi} v_{n}(\zeta)|d \zeta|
$$

for $\zeta \in C$ and $n=1,2, \cdots$, where $|d \zeta|$ denotes the linear measure on $C$. By using the Poisson formula

$$
v_{n}(z)=\int_{C} \frac{1-|z|^{2}}{|\zeta-z|^{2}} d_{\mu_{n}}(\zeta)
$$


for $z \in U$ and $n=1,2, \ldots$. In particular $\mu_{n}(C)=v_{n}(0)$ and thus by (4) $\left\{\mu_{n}(C)\right\}$ is bounded. In view of the selection theorem (see e.g. Constantinescu-Cornea $[2$, p. 9]), by choosing a suitable subsequence of $\left\{\mu_{n}\right\}$, we may assume that there exists a regular Borel measure $\mu_{\infty}$ on $C$ such that

$$
\lim _{n \rightarrow \infty} \int_{C} \lambda(\zeta) d \mu_{n}(\zeta)=\int_{C} \lambda(\zeta) d \mu_{\infty}(\zeta)
$$

for any real-valued continuous function $\lambda$ on $C$. By (4), (7) and (8) with $\lambda(\zeta)=\left(1-|z|^{2}\right) \cdot|\zeta-z|^{-2}$ we obtain

$$
v_{\infty}(z)=\int_{C} \frac{1-|z|^{2}}{|\zeta-z|^{2}} d \mu_{\infty}(\zeta)
$$

in $U$. The definition (6) of $\mu_{n}$ with (5) shows that $\mu_{n}(\beta)=0$ $(n=1,2, \cdots)$. Therefore by $(8)$ we conclude that

$$
\mu_{\infty}(\beta)=0 \text {. }
$$

Take a point $z_{1}$ in $\beta$ and let $\rho$ be the distance between $z_{1}$ and $C-\beta$. Then from (9) and (10) it follows that

$$
0<v_{\infty}(z)<4 \rho^{-2} \mu_{\infty}(C)\left(1-|z|^{2}\right)
$$

if $z \in U$ and $\left|z-z_{1}\right|<\rho / 2$. This shows that $v_{\infty}$ has continuous boundary value zero at $z_{1}$ and thus $v_{\infty}=0$ on $\beta$.

\section{REFERENCES}

1. R. Bader and M. Parreau, Domaines non compacts et classification des surfaces de Riemann, C. R. Acad. Sci. Paris 232 (1951), 138-139.

2. C. Constantinescu and A. Cornea, Ideale Ränder Riemannscher Flächen, Springer-Verlag, Berlin, 1963.

3. R. S. Martin, Minimal positive harmonic functions, Trans. Amer. Math. Soc. 49 (1941), 137-172.

4. A. Mori, On the existence of harmonic functions on a Riemann surface. J. Fac. Sci. Univ. Tokyo. 6 (1951), 247-257.

5. M. Parreau, Sur les moyennes des fonction harmoniques et analytiques et la classification des surfaces de Riemann, Ann. Inst. Fourier (Grenoble) 3 (1951), 103-197.

University of California, Los Angeles and Nagoya University 